\title{
Double face of cytochrome $c$ in cancers by Raman imaging
}

\author{
H. Abramczyk ${ }^{\circledR}$, B. Brozek-Pluska \& M. Kopeć(i)
}

Cytochrome $c$ (Cyt $c)$ is a key protein that is needed to maintain life (respiration) and cell death (apoptosis). The dual-function of $\mathrm{Cyt} c$ comes from its capability to act as mitochondrial redox carrier that transfers electrons between the membrane-embedded complexes III and IV and to serve as a cytoplasmic apoptosis-triggering agent, activating the caspase cascade. However, the precise roles of Cyt $c$ in mitochondria, cytoplasm and extracellular matrix under normal and pathological conditions are not completely understood. To date, no pathway of $\mathrm{Cyt} c$ release that results in caspase activation has been compellingly demonstrated in any invertebrate. The significance of mitochondrial dysfunctionality has not been studied in ductal carcinoma to the best of our knowledge. We used Raman spectroscopy and imaging to monitor changes in the redox state of the mitochondrial cytochromes in ex vivo surgically resected specimens of human breast tissues, and in vitro human breast cells of normal cells (MCF 10A), slightly malignant cells (MCF7) and highly aggressive cells (MDA-MB-231). We showed that Raman imaging provides insight into the biology of human breast ductal cancer. Here we show that proper concentration of monounsaturated fatty acids, saturated fatty acids, cardiolipin and Cyt $c$ is critical in the correct breast ductal functioning and constitutes an important parameter to assess breast epithelial cells integrity and homeostasis. We look inside human breast ducts by Raman imaging answering fundamental questions about location and distribution of various biochemical components inside the lumen, epithelial cells of the duct and the extracellular matrix around the cancer duct during cancer development in situ. Our results show that human breast cancers demonstrate a redox imbalance compared to normal tissue. The reduced cytochrome $c$ is upregulated in all stages of cancers development. The results of the paper shed light on a largely noninvestigated issues regarding cytochromes and mitochondrial function in electron transfer chain. We found in histopathologically controlled breast cancer duct that Cyt c, cardiolipin, and palmitic acid are the main components inside the lumen of cancerous duct in situ. The presented results show direct evidence that Cyt $c$ is released to the lumen from the epithelial cells in cancerous duct. In contrast the lumen in normal duct is empty and free of Cyt $c$. Our results demonstrate how Cyt $c$ is likely to function in cancer development. We anticipate our results to be a starting point for more sophisticated in vitro and in vivo animal models. For example, the correlation between concentration of Cyt $c$ and cancer grade could be tested in various types of cancer. Furthermore, Cyt $c$ is a target of anti-cancer drug development and a well-defined and quantitative Raman based assay for oxidative phosphorylation and apoptosis will be relevant for such developments.

Recent years have yielded exciting findings in the field of cancer cell metabolism, suggesting that change in the cellular redox status is important cancer driver, controlling various aspects of malignant progression ${ }^{1-13}$. Altered mitochondrial metabolism and redox state of cytochrome $c$ (Cyt $c$ ) is being increasingly recognized as an important factor, triggering various processes in cancer development ${ }^{5,6,13-17}$. It has been more than two decades since the central role of Cyt $c$ in mitochondrial pathway has been reported ${ }^{1,5,6,14-17}$. However, key issues regarding how Cyt $c$ is released from mitochondria and from cells still remain largely unclear ${ }^{3,5,6,14-17}$.

Cyt $c$ belongs to family of heme containing metalloproteins. Cyt $c$ is located in the intermembrane space of mitochondria and released into bloodstream during pathological conditions. Circulating in blood Cyt $c$ level is suggested to be a novel in vivo marker of mitochondrial injury after resuscitation from heart failure and chemotherapy ${ }^{18}$. Various existing techniques such as enzyme-linked immunosorbent assays (ELISA), Western blot, high performance liquid chromatography (HPLC), spectrophotometry and flow cytometry have been used to estimate Cyt $c$ concentration. However, the implementation of these techniques at POC (point of care) application is limited due to longer analysis time, expensive instruments and expertise needed for operation ${ }^{18}$. 
Moreover, none of the methods used to control Cyt $c$ concentration can provide direct evidence about the role of cytochrome $c$ in apoptosis and oxidative phosphorylation, because they are not able to monitor the amount of cytochrome in specific organelles such as mitochondria, cytoplasm, or extracellular matrix.

Here we show that Raman spectroscopy and Raman imaging are a promising label-free methods to estimate not only the level of Cyt $c$ in fast analysis of clinical practice, but also to identify localization and biochemical content in epithelial cells of the duct and in the extracellular matrix.

Until now, no technology has proven effective for detecting Cyt $c$ concentration in specific cell organelles. Therefore, existing analytical technologies cannot detect the full extent of Cyt $c$ localization inside and outside specific organelles. In Raman imaging we do not need to disrupt cells to break open the cells and release the cellular structures to learn about their biochemical composition.

Cyt $c$ is not only serving as an cell death biomarker (apoptosis), but is also a key protein that is needed to maintain life (respiration). Thus, it is of great importance to understand the role of Cyt $c$ in certain diseases at cellular level ${ }^{6}$. Here we will concentrate on breast cancer.

Here we show that mitochondrial content of Cyt $c$ is critical in the correct breast ductal functioning and constitutes an important parameter to assess breast epithelial cells integrity and homeostasis. We look inside human breast ducts answering fundamental questions about location and distribution of various biochemical components inside the lumen, epithelial cells of the duct and the extracellular matrix around the cancer duct during cancer development in situ.

We studied oncogenic processes that characterize human breast cancer (ductal cancer in situ (DCIS) and infiltrating ductal carcinoma (IDC)) based on the quantification of cytochrome redox status by exploiting the resonance-enhancement effect of Raman scattering.

In this paper we explore a hypothesis involving the role of reduction-oxidation pathways related to Cyt $c$ in cancer development. Here we show that Raman spectroscopy and Raman imaging are competitive clinical diagnostics tools for cancer diseases linked to mitochondrial dysfunction and are a prerequisite for successful pharmacotherapy of cancer.

\section{Results}

To properly address redox state changes of mitochondrial cytochromes in breast cancers by Raman spectroscopy and imaging, we systematically investigated how the Raman method responds to in vitro human cells and ex vivo human tissues. In vitro experiments will allow to study a single cell by reducing the role of cell-to cell interactions. The ex vivo human tissue experiments will extend our knowledge on the influence of environment on cancer development.

Figure 1 shows the cross section through the normal breast duct obtained by Raman imaging. Details of the experimental method used to create Raman image are given in section "Materials and methods". The Raman image is compared with the microscopy image. The characteristic vibrational spectra for different areas of the breast tissue are also presented in Fig. 1. One can see from Fig. 1 that there is an almost perfect match between the morphological features and Raman images. However, Raman imaging provides additional information, which is not available from histology, microscopy, mammography, and fluorescence. It is biochemical information. To understand biochemical information that is provided from Raman images we need to associate the characteristic features with the breast morphology. Briefly, the normal organization of ducts demonstrates lumen surrounded by epithelial cells aligned in a polar manner so their apical side faces the lumen. These cells are surrounded by the basement membrane. The next layers represents extracellular matrix consisting of fibroblasts and the stroma, which is predominantly, but not exclusively, composed of connective tissue and adipose tissue. Schematic structures of epithelial tissue, stromal and adipocyte cells around the normal breast duct are presented in Scheme 1.

Comparing Raman and microscope images from Fig. 1 with the Scheme 1 it is easy to identify all morphological features of the normal duct.

One can see that yellow-blue line around the black duct represents normal epithelial cells that are lined along the intact basement membrane and do not proliferate inside the lumen and outside through the basement membrane. Raman images provides information not only about morphology, but also about biochemistry of these structures that is given by the Raman spectra in Fig. 1.

The Raman spectra presented in Fig. 1 show the biochemical composition of the structures. One can see from Fig. $1 \mathrm{~B}$ that the lumen is empty (black colour) with no Raman spectra indicating that there are no epithelial/ mesenchymal cells inside the normal duct. The epithelial cells of the normal duct contain oleic acid, $\beta$-carotene, cardiolipin, palmitic acid. The epithelial cells are dominated by monounsaturated oleic acid derivatives composed of glyceryl trioleate and carotenoids (Fig. 1F). Indeed, the characteristic Raman vibrations of carotenoids with resonance peaks at 1156 and $1526 \mathrm{~cm}^{-1}$ are clearly visible in Fig. 1F. The peaks at 2852, 2928, $3009 \mathrm{~cm}^{-1}$ correspond to the vibrations of monounsaturated oleic acid ${ }^{19-21}$. Figure S1 in Supplementary Materials shows comparison of average Raman spectra obtained by Cluster Analysis Method and the Raman spectra characteristic for pure chemical components. To show the perfect match between Raman spectra of carotenoids and monounsaturated oleic acid derivatives in human normal duct and Raman spectra of pure isolated compounds the correlation analysis was performed (Pearson correlation coefficient was equal 1.0 at the confidence level 0.95), see Table 1 in Supplementary Materials and Pearson correlation coefficient for all components.

The extracellular matrix (red colour in Fig. 1C) is dominated by collagen (see Fig. S1 in Supplementary Materials). Small concentration of oxidized cytochrome $c\left(\mathrm{Fe}^{3+}\right.$ green colour in Fig. 1) was found with a characteristic Raman peaks at 750,1126, $1582 \mathrm{~cm}^{-1}$ and $1634 \mathrm{~cm}^{-122}$. The oxidized form of cytochrome $c\left(\mathrm{Fe}^{3+}\right)$ can induce caspase activation in the process of apoptosis, while the reduced form $\left(\mathrm{Fe}^{2+}\right)$ cannot $^{23}$. The oxidized cytochrome $c\left(\mathrm{Fe}^{3+}\right)$ is not bound to cardiolipin and can participate in electron shuttling of the respiratory chain and in oxidative phosphorylation ${ }^{24}$. Cardiolipin, is abundantly present in mitochondria in the inner mitochondrial 
H

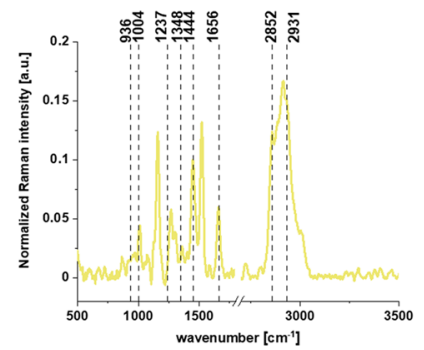

Epithelial cells Mammaglobin-A

\section{Extracellular \\ Matrix \\ Cytochrome C \\ $\beta$-Carotene, \\ Lipids}
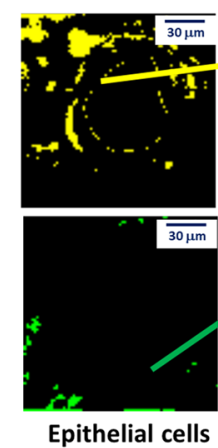

pithelial cells

Oleic acid

$\beta$-caroten

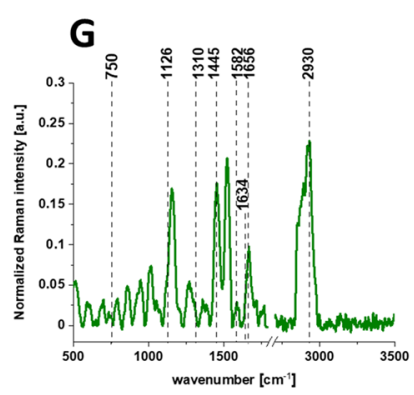

\section{$F$}

A

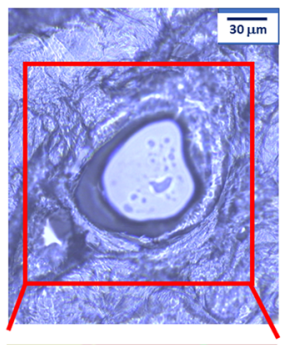

B

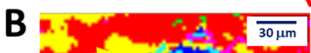

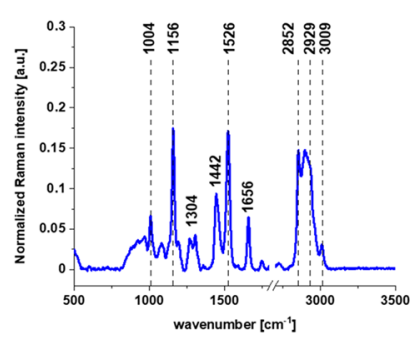

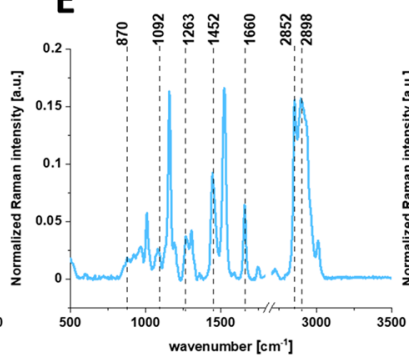

동ำ

C

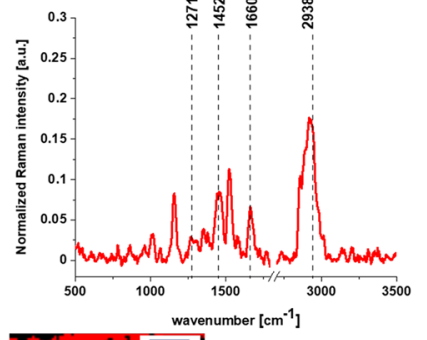

Extracellular

Matrix

Collagen

Extracellular

Matrix

Palmitic acid

D

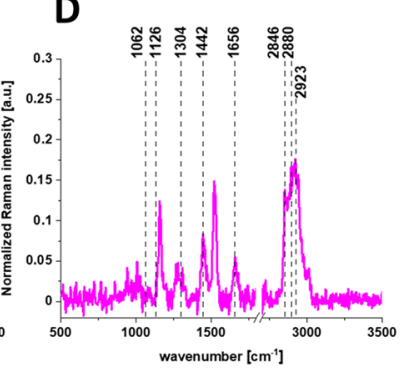

Figure 1. (A) Microscopy image of human normal duct, (B) Raman imaging of human normal duct obtained by using Cluster Analysis and Raman images and average Raman spectra (normalized by norm) of all clusters identified by Cluster Analysis: (C) collagen (red), (D) palmitic acid (pink), (E) cardiolipin (turquoise), (F) oleic acid, (G) (blue), cytochrome $c$ (green), (H) mammaglobin-A (yellow).

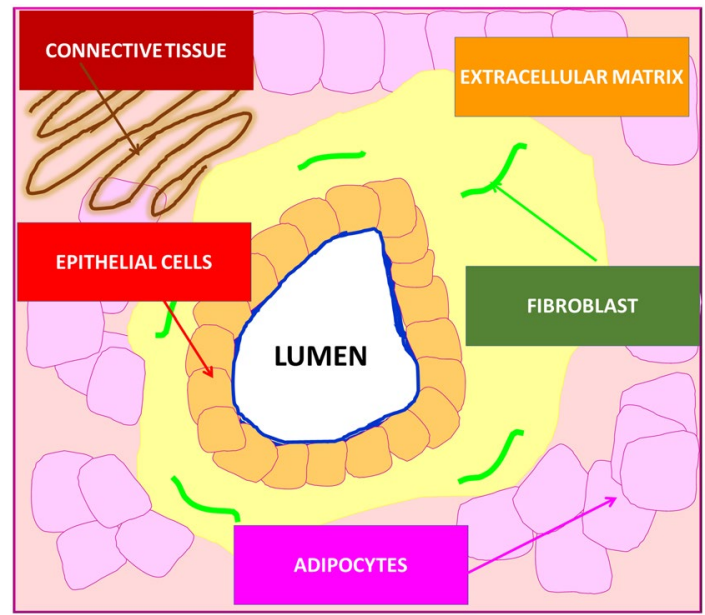

Scheme 1. Schematic representation of the structure of human normal duct. 


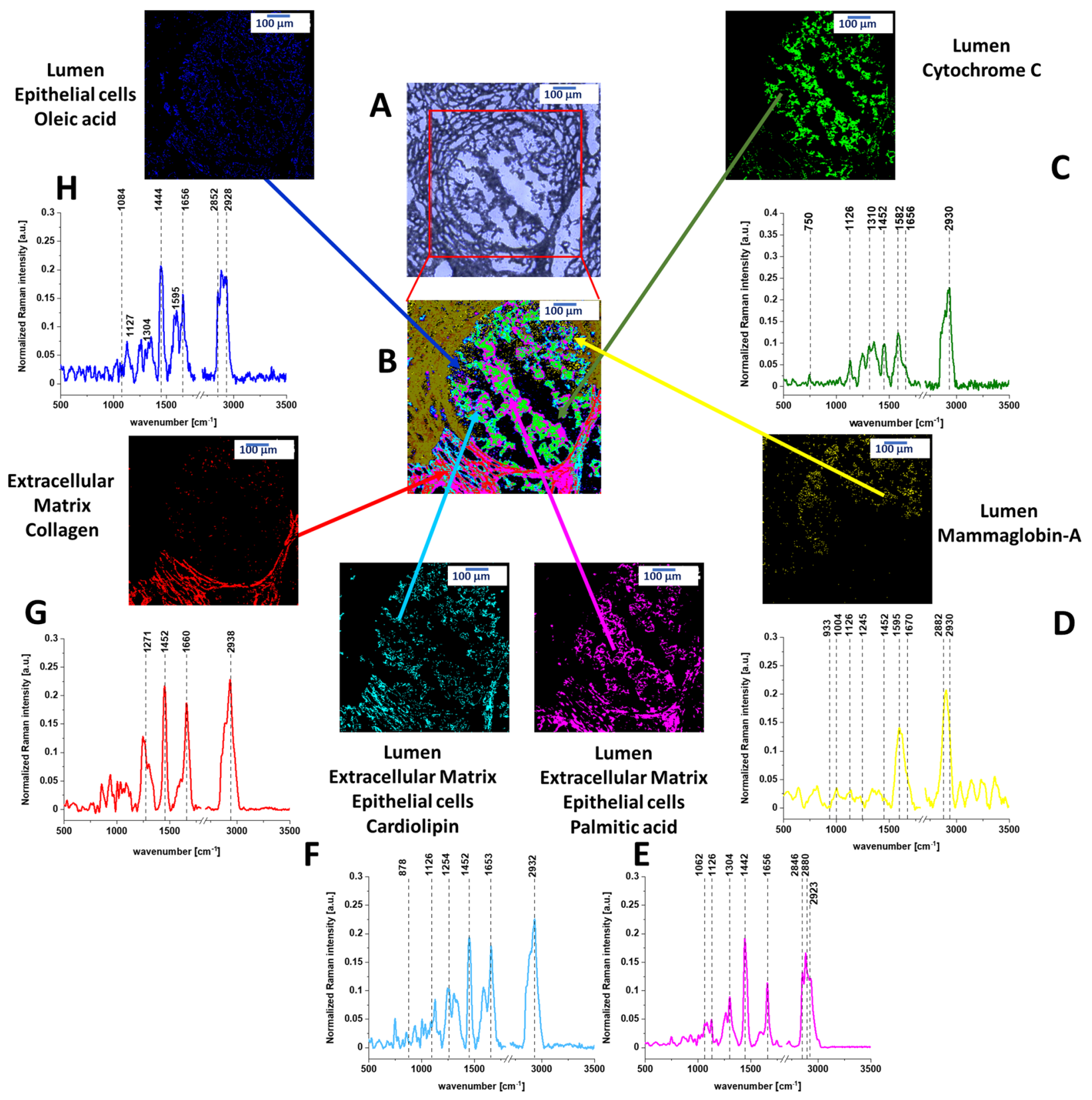

Figure 2. (A) Microscopy image of human cancerous duct, (B) Raman imaging of human cancerous duct obtained by using Cluster Analysis and Raman images and average Raman spectra (normalized by norm) of all clusters identified by Cluster Analysis: (C) cytochrome $c$ (green), (D) mammaglobin-A (yellow), (E) palmitic acid (pink), (F) cardiolipin (turquoise), (G) collagen (red), (H) oleic acid (blue).

membrane, where it constitutes about $20 \%$ of the total lipid composition ${ }^{17}$. The band at $1656 \mathrm{~cm}^{-1}$ represents Amide I vibration of proteins and $\mathrm{C}=\mathrm{C}$ stretching vibration of unsaturated lipids (mainly monounsaturated oleic acid) $)^{19,25}$.

In contrast to the normal duct in Fig. 1, the cancerous duct presented in Fig. 2 shows that the normal organization of the epithelial cells is lost and the lumen is filled with the cancerous cells. It would be extremely interesting to learn what chemical substances are released from the epithelial cells into the lumen during cancer development, because monitoring biochemical alterations would drive the progress on mechanisms of cancer to limits just unimaginable a few years ago.

Figure 2 shows the Raman image of the cross section through the cancerous duct. Figure 2 demonstrates that the biochemical profile of the lumen of the cancerous duct contains four main components: cytochrome $c$ (green colour), cardiolipin (turqoise), palmitic acid (pink colour) and mammaglobin-A (yellow colour) (Details are presented in Fig. S2 of Supplementary Materials) in contrast to the lumen in the normal duct which is empty (Fig. 1). The cytochrome $c$ represents the reduced form $\left(\mathrm{Fe}^{2+}\right)$ as the Raman signal at $1582 \mathrm{~cm}^{-1}$ of the reduced form is an order higher than that of the oxidized form $\left(\mathrm{Fe}^{3+}\right)$ (see Supplementary Materials). The Raman spectrum of Cyt $c$ in Fig. 2 shows that Cyt $c$ is bound to a lipid identified as a cardiolipin $\left(1452 \mathrm{~cm}^{-1}\right)$. The band at 
$1452 \mathrm{~cm}^{-1}$ of cardiolipin does not overlap with the C-H deformation bands of saturated lipids of palmitic acid at $1442 \mathrm{~cm}^{-1}$. In addition, one can see that the Raman intensity of the band at $1656 \mathrm{~cm}^{-1}$ in Fig. $2 \mathrm{C}$ corresponding to $\mathrm{C}=\mathrm{C}$ vibrations of unsaturated lipids is much lower than that of reduced cytochrome $c$ at $1582 \mathrm{~cm}^{-1}$ in contrast to the normal duct (Fig. $1 \mathrm{G}$ ). This is a result of decrease of $\mathrm{C}=\mathrm{C}$ vibration from monounsaturated oleic acid, which is evident from comparison Raman signals at $1656 \mathrm{~cm}^{-1}$ between Figs. $1 \mathrm{~F}$ and $2 \mathrm{H}$.

Cyt $c$ is mostly protonated meaning that most Cyt $c$ bonds via electrostatic bonds to acidic phospholipids, particularly cardiolipin. Cardiolipin-bound Cyt $c$, probably does not participate in electron shuttling of the respiratory chain ${ }^{24}$. It indicates that the process of oxidative phosphorylation (respiration) becomes less effective in cancer cells (known as Wartburg effect).

On the other hand, the reduced form of cytochrome $c\left(\mathrm{Fe}^{2+}\right)$ cannot induce caspase activation and the process of apoptosis in cancerous cells becomes less efficient ${ }^{23}$.

Figures 1 and 2 were obtained by Cluster Analysis (CA). To identify the chemical compounds we compared the Cluster Analysis spectra with the following components: oleic acid, $\beta$-carotene, palmitic acid, mammaglobinA, collagen, cytochrome $c$, cardiolipin. Figures S1 and S2 in the Supplementary Materials show comparison between the Raman spectra of the chemical components with CA Raman spectra. Table 1 from Supplementary Materials presents the Pearson correlation coefficients obtained for comparison of the average Raman spectra typical for normal and cancerous human duct and the Raman spectra characteristic for pure components such as: oleic acid, $\beta$-carotene, palmitic acid, mammaglobin-A, collagen, cytochrome $c$, cardiolipin. The Pearson correlation coefficients demonstrates the perfect agreement between CA and Raman spectra of isolated chemical components.

To estimate distribution and concentration of the chemical components in the normal and cancerous duct we applied Basis Analysis (BA). The detailed results of BA of normal and cancerous ducts are presented in Supplementary Materials (Figs. S3 and S4). During the BA analysis each measured spectrum of the 2D spectral array of the analyzed human breast sample was compared to the spectra of pure chemical components mentioned above using a least square to fit each convergence to minimize the fitting error. BA method confirms the most important features of biodistribution in normal and cancerous ducts. First, $\beta$-carotene is present in abundant amount in the epithelial cells of the normal duct, while is absent in cancerous duct (blue colour in Fig. S3). Second, small amount of oxidized Cyt $c$ is localized in extracellular matrix (green colour) in Fig. S3 and large amount of reduced cytochrome $c$ is observed in the epithelial cells, lumen and extracellular matrix in the cancerous duct (Fig. S4). Third, localization of cardiolipin and cytochrome $c$ is drastically different in normal duct: cardiolipin is almost exclusively localized in the epithelial cells (turquoise colour in Fig. S3) in contrast to Cyt $c$, which is localized in small amount in the extracellular matrix. It indicates that cytochrome $c$ and cardiolipin are not bound due to different areas of localization. In contrast, in the cancerous ducts the areas of localization are overlapped in the region of lumen, epithelial cells and extracellular matrix (Fig. S4). It means that cytochrome $c$ and cardiolipin may interact and be bound due to electrostatic interactions.

Detailed inspection into the Raman intensities of different components (presented by the bars at left side of each distribution in Figs. S3 and S4) allows to analyzing the lipid profile. The lipid profile of the lumen in the cancerous breast duct in Fig. S4 is dominated by a mixture of cardiolipin (turquoise colour), palmitic acid (pink colour) and oleic acid (blue colour) with no presence of carotenoids in contrast to normal epithelial cells in the normal duct filled with monounsaturated oleic acid derivatives composed of glyceryl trioleate and carotenoids (Fig. S3). We found that the ratio between the monounsaturated oleic acid and palmitic acid is 3:1 in normal duct and $1: 1$ in cancerous duct. We found also that the ratio between cytochrome $c$ in normal duct and in cancerous duct is 1:47 and we noticed that the ratio between cardiolipin in normal duct and in cancerous duct is 1:1.6.

The alterations in the lipid composition in the epithelial cells must have very serious consequences. Incorporation of saturated lipids, such as cardiolipin and palmitic acid into lipid membranes is known to stiffen a membrane. Such membranes can be described as "a rigid amorphous glass state" 24 leading to distortions and deformations. The extracellular matrix around the cancerous duct is dominated by a network consisting of complementary regions of collagen (red colour) and other proteins (mammaglobin-A, yellow colour). Strong fluorescence at $599 \mathrm{~nm}$ (yellow dark colour in the upper left corner in Fig. 2B) for the excitation at $532 \mathrm{~nm}$ is also observed.

First we will concentrate on contribution of cytochrome $c$ to the cancerous duct. Figure 1 shows that in normal duct, Cyt $c$ is located around the epithelial cells in the extracellular matrix. Figure 2 shows that in the cancerous duct Cyt $c$ is located in the lumen. To show the perfect match between Raman spectra in the lumen of the human cancerous duct and Raman spectrum of isolated Cyt $c$ the correlation analysis was performed (Pearson correlation coefficient was equal 1.0 at the confidence level 0.95), see Supplementary Materials Table 1.

Therefore, the results in Fig. 2 provides the first direct evidence for the release of Cyt $c$ from epithelial cells into the lumen of the cancerous duct in situ where it has a reduced form. The mechanisms how Cyt $c$ is released to the lumen of the cancerous duct is still unknown, but in the view of the presented results they must be related to the lipid composition of the epithelial cells.

At normal physiological conditions, Cyt $c$ is located in the mitochondrial intermembrane/intercristae spaces of cells, where it functions as an electron shuttle in the respiratory chain and interacts with cardiolipin ${ }^{17}$. It is commonly believed that proapoptotic oncogenic stimuli induce the permeabilization of the outer membrane allowing for Cyt $c$ release to cytosol. In the cytosol, Cyt $c$ mediates the allosteric activation of apoptosis-protease activating factor 1 , which is required for the proteolytic maturation of caspase- 9 and caspase-3. Activated caspases ultimately lead to apoptotic cell dismantling ${ }^{17}$. There are a few possible scenarios of the outer membrane permeabilization such as induction of mitochondrial permeability transition, Bcl-family proteins and mitochondrial outer membrane permeabilization, Volume-dependent, MPT-independent mechanisms of cytochrome $c$ release, caspase-2-mediated release of Cyt $c^{5}$. 
Our results suggest that permeability of the membranes is simply related to the modifications of their biochemical composition of lipids during cancer development. We found that the protein/lipid profiles inside the lumen and in the epithelial cells of the cancerous duct are markedly different for the normal and the cancerous ducts. Our results (Fig. 1) demonstrates that the epithelial cells of the normal duct are dominated by monounsaturated fatty acids that contributes to a proper membrane fluidity of the epithelial cells. The cardiolipin and palmitic acid are located around the epithelial cells in the extracellular matrix. In contrast, the epithelial cancerous cells and the lumen are enriched with saturated lipids (cardiolipin and palmitic acid). Membranes of cells are the primary target for injury and their damage and are highly dependent on their physical properties and lipid organization. The abnormal proportion between saturated and unsaturated fatty acids that we found in the epithelial cells of the cancerous duct (Fig. 2) effects fluidity of the membranes leading to distortions and deformations and decrease of mechanical stability ${ }^{24,26-29}$. Membrane fluidity is a key property for maintaining cell functionality, and depends on lipid composition and cell environment ${ }^{29}$. The effects of mechanical deformations due to modifications of fluidity result in expelling cardiolipin, Cyt $c$ and palmitic acid into the lumen of the cancerous duct. The distortion of the epithelial cells allows Cyt $c$ to be released to lumen.

In the view of the results presented so far one can propose discrete sequence of biochemical events that lead to malignant transformation of the epithelial cells in the normal breast duct. First, the upregulation of glycolysis ${ }^{30-35}$ leads to enhanced synthesis of fatty acids de novo ${ }^{36-42}$. De novo fatty acids synthesis changes biochemical composition of the epithelial cells as one can see from the comparison of Figs. 1 and 2. The altered fluidity of the membranes of epithelial cells leads to mechanical deformations allowing for Cyt $c$ release to lumen of the duct. In this hypothesis the release of Cyt $c$ is a result, not a cause, of malignant transformation due to lipid reprogramming in cancer development ${ }^{22,43,44}$. To date, no pathway of Cyt $c$ release that results in caspase activation has been compellingly demonstrated in any invertebrate, despite the presence of homologues of many of the molecules that mediate and/or regulate the intrinsic pathway of apoptosis in vertebrate cells. Furthermore, little is known about the extent of Cyt $c$ release (if any) in cells that do not die ${ }^{6}$.

We anticipate our results presented so far to be a starting point for more sophisticated analysis of statistical significance. That is why we tested the correlation between concentration of Cyt $c$ and cancer grade in ductal breast cancer for a number of patients $n=39$.

Recently we reported ${ }^{22,45}$ the results for normalized by norm Raman intensity of the characteristic vibration of cytochrome $c$ and $\mathrm{b}\left(750,1126,1337\right.$ and $\left.1584 \mathrm{~cm}^{-1}\right)$ as a function of grade for human breast normal (G0) and cancer tissues (G1, G2, G3). The normalized intensities have many advantages as discussed in the previous papers $^{22,45}$. However, only the absolute Raman intensities are directly proportional to concentrations and are more useful for the purposes of this paper. We performed detailed statistical analysis (one-way ANOVA) for $\mathrm{n}=39$, for each patient thousands spectra (typically 6400) obtained from Raman imaging were used for averaging. Based on the average values obtained for the Raman biomarkers of Cyt $c$ and b we obtained a plot as a function of ductal cancer breast grade malignancy for human in situ and infiltrating ductal carcinoma (IDC) compared with a control group (normal tissue without ductal cancer) presented in Fig. 3.

One can see from Fig. 3 that the global concentration of cytochrome $c$ in the breast tissue (reflected by the Raman intensity of the bands at $1584 \mathrm{~cm}^{-1}$ and $750 \mathrm{~cm}^{-1}$ ) increases with cancer aggressiveness. The results from Fig. 3 demonstrate that the concentration of Cyt $c$ is upregulated in breast cancer cells.

The correlation between Cyt $c$ concentration and cancer aggressiveness is characterized by gradually increasing Raman signal at $1584 \mathrm{~cm}^{-1}$ indicating progressive redox state changes and supports the results for the normalized Raman spectra of cytochrome $c$ in the ductal cancer of breast tissues ${ }^{22}$.

To understand the results presented in Fig. 3 we need to examine single normal and cancerous cells in vitro, where we will be able to directly monitor the concentration of cytochrome in separate organelles of the epithelial cells: mitochondria, cytosol, lipid droplets and nucleus by using Raman imaging.

Let us concentrate on Cyt $c$ concentration in mitochondria in breast cancer cells. We studied human epithelial breast cells of the malignant cells (MCF7). Cluster Analysis results for a single human epithelial breast cancerous cell of MCF7 (G2) are presented in Supplementary Materials in Fig. S5.

We visualized localization of cytochromes by Raman imaging in the major organelles of cells. We demonstrated that the "redox state Raman marker" of the ferric low spin heme in Cyt $c$ at $1584 \mathrm{~cm}^{-1}$ can serve as a sensitive indicators of cancer aggressiveness.

To summarize, our results show that the concentration of Cyt $c$ increases with cancer aggressiveness. It indicates that the netto concentration of Cyt $c$ in mitochondria is higher than release to cytoplasm. This finding reflects the dual face of Cyt in life and death decisions: apoptosis and oxidative phosphorylation. The balance between cancer cells proliferation (oxidative phosphorylation) and death (apoptosis) decide about level of cancer development. The Cyt $c$ concentration in mitochondria as a function of cancer aggressiveness reflects its contribution to oxidative phosphorylation and apoptosis ${ }^{4}$. Normal cells (G0) primarily produce energy through glycolysis followed by mitochondrial citric acid cycle and oxidative phosphorylation.

We proved that the signal at $1584 \mathrm{~cm}^{-1}$ for normal epithelial cells (G0) represents predominantly the oxidized form $\left(\mathrm{Fe}^{3+}\right)$ of Cyt $c$ unbound to cardiolipin. It indicates that both apoptosis can be induced and electron shuttling between the complex III, Cyt $c$, and complex IV can occur leading to effective oxidative phosphorylation (respiration). In contrast, for cancerous cells $(\mathrm{G} 2, \mathrm{G} 3)$ the concentration of reduced form $\left(\mathrm{Fe}^{2+}\right)$ of Cyt $c$ bound to cardiolipin increases. Cardiolipin-bound Cyt $c$, probably does not participate in electron shuttling of the respiratory chain ${ }^{29}$, and reduced cytochrome cannot induce caspase and apoptosis process.

Our results demonstrate that cancer cells produce their energy predominantly through the oxidative phosphorylation, in contrast to Warburg hypothesis that most cancer cells produce their energy through a high rate of glycolysis followed by lactic acid fermentation even in the presence of oxygen. However, the effectiveness of the oxidative phosphorylation decreases with cancer aggressiveness due to higher concentration of cardiolipin bound to Cyt $c$. 

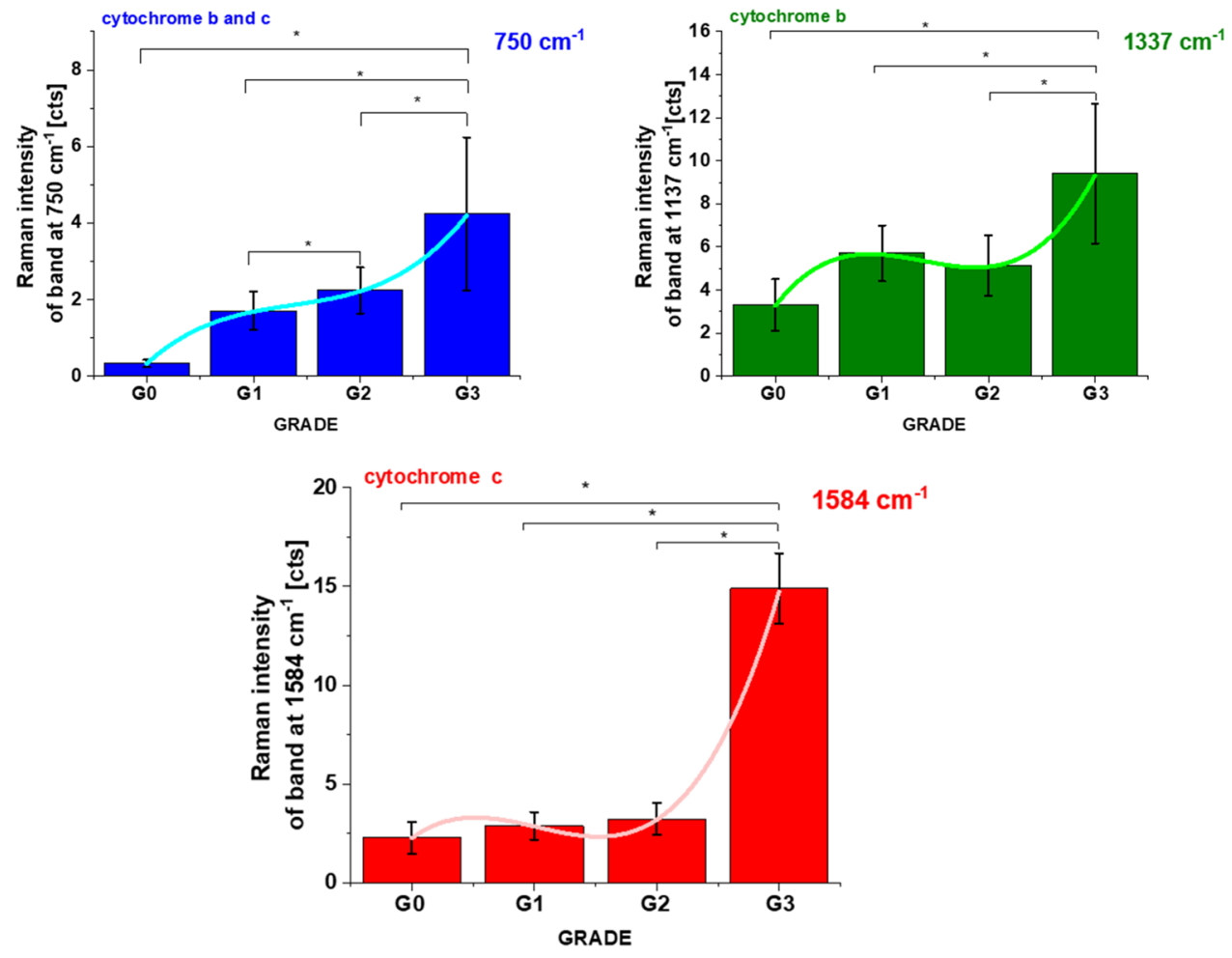

Figure 3. The Raman intensities $\mathrm{I}_{750}, \mathrm{I}_{1337}, \mathrm{I}_{1584}$ of Cyt $c$ and Cyt $\mathrm{b}$ bands in human breast tissues as a function of breast cancer grade malignancy G0-G3 at excitation $532 \mathrm{~nm}$, average \pm SD, the statistically significant result have been marked with asterisk. The one-way ANOVA using the Tukey test was used to calculate the value significance, asterisk ${ }^{*}$ denotes that the differences are statistically significant, $p$-values $\leq 0.05$ were accepted as statistically significant.

Our results presented in this paper provide experimental evidence on the role of cytochrome $c$ in cancer development via the following mechanism: in normal cells Cyt $c$ is localized predominantly in the mitochondria. The release of Cyt $c$ into the cytoplasm is believed to induce the non-inflammatory process of apoptosis ${ }^{5,17}$. When it is transferred to the extracellular space, it can cause inflammation and cancer development. The assessment of Cyt $c$ in the extracellular space might be used as a biomarker for determine mitochondrial damage and cell death.

To estimate the concentration of cytochrome $c$ we have performed the reference curves for correlation between the Raman intensities of oxidized and reduced forms of Cyt $c$ and their concentrations. Figure 4 shows the intensity of Raman peak centered at $1584 \mathrm{~cm}^{-1}$ as a function of Cyt $c$ concentration for oxidized and reduced forms for experimental conditions used for cells (Fig. 4A,C) and tissues measurements (Fig. 4B,D).

One can see that we do not observe the linear dependence of Raman intensities and the Cyt $c$ concentration. These results are expected because the characteristic Raman intensities in the resonance Raman effect are not directly proportional to the concentrations of the compounds and deviate due to absorption resonance effects at $532 \mathrm{~nm}$ excitation (Q band electron absorption of Cyt $c$ ).

The levels of 2.3 for G0 and 14.9 for G3 for Raman intensities obtained for human breast tissues in Fig. 3 correspond to the Cyt $c$ concentrations of $19 \mu \mathrm{M}$ (based on Fig. 4B) and $266 \mu \mathrm{M}$ (based on Fig. 4D), respectively.

The high differences between concentration of Cyt $c$ in normal (G0) and cancerous (G3) tissues results from the extracellular matrix contribution. In the tissue the combined effect of localization in mitochondria, the release of cytochrome $c$ from mitochondria into the cytoplasm and from cytoplasm into extracellular space is monitored. The results of this paper indicate that the release of Cyt $c$ to extracellular space is the critical mechanism in the process of cancer development.

To summarize, the results provide evidence on the prominent role of cytochrome $c$ in the intrinsic pathway of apoptosis and oxidative phosphorylation vs cancer aggressiveness. It is the first report providing direct evidence by Raman imaging on the role of cardiolipin-Cyt $c$ complex on apoptosis and oxidative phosphorylation both for epithelial cells of ducts in the tissue and cells in vitro. Moreover, the paper provides the method to estimate the concentration of Cyt $c$ from the absolute Raman intensities of the oxidized and reduced Cyt $c$ in tissues and single cells. Until now, no technology has proven effective for detecting Cyt $c$ concentration in specific cell organelles. Therefore, existing analytical technologies cannot detect the full extent of Cyt $c$ localization inside and outside specific organelles. In Raman imaging we do not need to disrupt cells to break open them and release the cellular structures to learn about their biochemical composition.

Nevertheless, there is still much to learn. Here we demonstrate that Raman imaging reveal new expanses on the role of Cyt $c$ in cancer biology and mechanisms of apoptosis and oxidative phosphorylation. We anticipate 
A

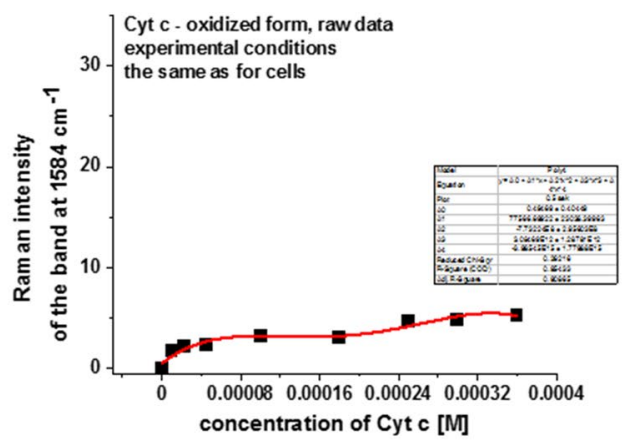

C

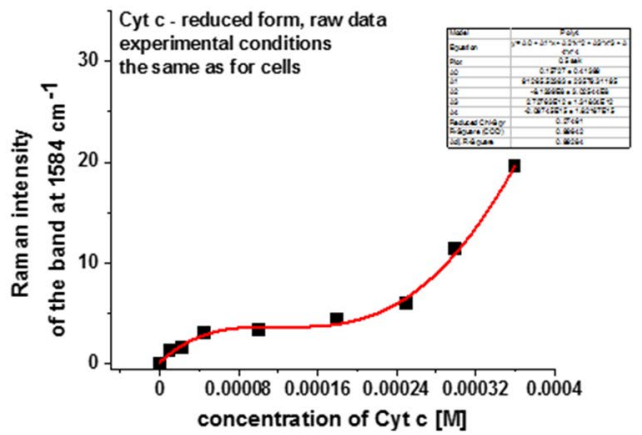

B

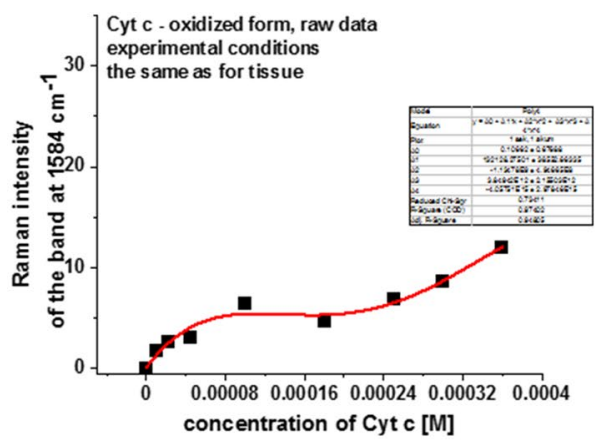

D

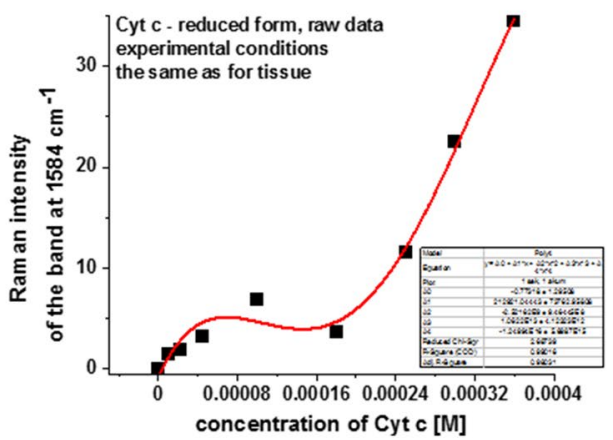

Figure 4. The intensity of Raman peak centered at $1584 \mathrm{~cm}^{-1}$ as a function of Cyt $c$ concentration (raw data) for: (A) the oxidized form of Cyt $c$, experimental conditions the same as for breast single cells measurements: integration time $0.5 \mathrm{~s}, 1$ accumulation, laser power $10 \mathrm{~mW}$; (B) the oxidized form of Cyt $c$, experimental conditions the same as for breast tissue measurements: integration time $1.0 \mathrm{~s}, 1$ accumulation, laser power 10 $\mathrm{mW}$; (C) the reduced form of Cyt $c$, experimental conditions the same as for breast single cells measurements: integration time $0.5 \mathrm{~s}, 1$ accumulation, laser power $10 \mathrm{~mW}$, reduction agent $\mathrm{NaBH}_{4}$ in tenfold excess; (D) the reduced form of Cyt $c$, experimental conditions the same as for breast tissue measurements: integration time $1.0 \mathrm{~s}, 1$ accumulation, laser power $10 \mathrm{~mW}$, reduction agent $\mathrm{NaBH}_{4}$ in tenfold excess.

our results to be a starting point for more sophisticated ex vivo human tissues, in vitro and in vivo animal models. For example, the correlation between concentration of Cyt $c$ and cancer grade could be tested in various types of cancer. Furthermore, Cyt $c$ is a target of anti-cancer drug development ${ }^{10-12}$ and a well-defined and quantitative Raman based assay for oxidative phosphorylation and apoptosis will be relevant for such developments.

\section{Materials and methods}

Reagents. All reagents were purchased from Sigma Aldrich (Poland) unless otherwise stated. Cytochrome $c$ (no. C2506), cardiolipin (no. C0653).

Ethics statement. All experiments were performed in accordance with relevant guidelines and regulations of the Bioethical Committee at the Medical University of Lodz, Poland (RNN/323/17/KE/17/10/2017) and (RNN/18/20/KE). The experimental protocols were approved by Bioethical Committee at the Medical University of Lodz, Poland (RNN/323/17/KE/17/10/2017) and (RNN/18/20/KE). Written informed consent was obtained from all patients, or if subjects are under 18 , from a parent and/or legal guardian. All the experiments were carried out in accordance with Good Clinical Practice and with the ethical principles of the Declaration of Helsinki. Spectroscopic analysis did not affect the scope of surgery and course and type of undertaken hospital treatment.

Patients. In the presented studies the total number of patients diagnosed with breast cancer was 39 . All patients were diagnosed with ductal carcinoma in situ (DCIS) in situ or invasive ductal carcinoma and treated at the M. Copernicus Voivodeship Multi-Specialist Center for Oncology and Traumatology in Lodz.

Tissues samples collection and preparation for Raman spectroscopy. Tissue samples were obtained during routine surgery. The fresh, non-fixed samples were used to prepare $16 \mu \mathrm{m}$ sections placed on $\mathrm{CaF}_{2}$ substrate for Raman analysis. The adjacent slices were used for histopathological analysis, which was performed by professional pathologists from Medical University of Lodz, Department of Pathology, Chair of Oncology. The types and grades of cancers were diagnosed according to the criteria of the Current WHO by pathologists from Medical University of Lodz, Department of Pathology, Chair of Oncology. 
Subculture of cell lines. A human breast MCF10A cell line (CRL10317, ATCC) was grown with completed growth medium: MEGM Kit (Lonza CC3150) without gentamycin-amphotericin B mix (GA1000) and with $100 \mathrm{ng} / \mathrm{ml}$ cholera toxin; a slightly malignant human breast MCF7 cell line (HTB22, ATCC) in Eagle's Minimum Essential Medium (ATCC 30-2003) with 10\% fetal bovine serum (ATCC 30-2020) and highly aggressive human breast MDA-MB-231 cell line (HTB26, ATCC) in Leibovitz's L15 Medium (ATCC 30-2008) with 10\% fetal bovine serum (ATCC 30-2020). All human breast cell lines were maintained at $37^{\circ} \mathrm{C}$ in a humidified atmosphere containing $5 \% \mathrm{CO}_{2}$. Cells were seeded on $\mathrm{CaF}_{2}$ window in $35 \mathrm{~mm}$ Petri dish at a density of $5 \times 10^{4}$ cells per Petri dish the day before examination. Before Raman examination, cells were fixed with $4 \%$ formalin solution (neutrally buffered) and kept in phosphate buffered saline (PBS, Gibco no. 10010023) during the experiment.

Raman imaging for assessing cytochrome $c$ release in human tissues and in vitro cells. The status of Cyt $c$ (whether intact in the mitochondria or released) was examined by WITec (Ulm, Germany) alpha $300 \mathrm{RSA}+$ confocal microscope by recording Raman spectra and images. All images were acquired by the experimental set up consisting of diode laser $532 \mathrm{~nm}$, the fibre of $50 \mu \mathrm{m}$, a monochromator Acton-SP-2300i and a CCD camera Andor Newton DU970-UVB-353. The excitation line was focused on the sample through a $40 \times$ dry objective (Nikon, objective type CFI Plan Fluor C ELWD DIC-M, numerical aperture (NA) of 0.60 and a 3.6-2.8 mm working distance) for tissue measurements and $40 \times$ water dipping objective (Zeiss $\mathrm{W}$ planApochromat, VIS-IR, N numerical aperture (NA) of 1.0 and a $2.5 \mathrm{~mm}$ working distance) for human breast cell lines. The average laser excitation power was $10 \mathrm{~mW}$ for 532, with an integration time of $1.0 \mathrm{~s}$ for low frequency range and 0.5 for high frequency range. An edge filters were used to remove the Rayleigh scattered light. A piezoelectric table was used to record Raman images. The cosmic rays were removed from each Raman spectrum (model: filter size: 2, dynamic factor: 10) and the smoothing procedure: Savitzky-Golay method was also implemented (model: order: 4, derivative: 0 ). Data acquisition and processing were performed using WITec Project Plus software. The background subtraction and the normalization [model: divided by norm (divide the spectrum by the dataset norm)] were performed by using Origin software according to the formula:

$$
\begin{gathered}
V^{\prime}=\frac{V}{\|V\|} \\
\|V\|=\sqrt{v_{1}^{2}+v_{2}^{2}+\ldots v_{n}^{2}}
\end{gathered}
$$

where: $v_{n}$ is the nth $\mathrm{V}$ values.

The normalization was performed for low $\left(500-1800 \mathrm{~cm}^{-1}\right)$ and high $\left(2600-3500 \mathrm{~cm}^{-1}\right)$ frequency spectral regions separately.

Cluster analysis. Spectroscopic data were analyzed using Cluster Analysis method. Briefly Cluster Analysis is a form of exploratory data analysis in which observations are divided into different groups that have some common characteristics-vibrational features in our case. Cluster Analysis constructs groups (or classes or clusters) based on the principle that: within a group the observations must be as similar as possible, while observations belonging to different groups must be different.

The partition of $\mathrm{n}$ observations $(\mathrm{x})$ into $\mathrm{k}(\mathrm{k} \leq \mathrm{n})$ clusters $\mathrm{S}$ should be done to minimize the variance (Var) according to the formula:

$$
\arg \min _{s} \sum_{i=1}^{k} \sum_{x \in S i}\left\|x \mu_{i}\right\|^{2}=\arg \min _{s} \sum_{i=1}^{k}\left|S_{i}\right| \operatorname{Var} S_{i}
$$

where $\mu_{i}$ is the mean of points $S_{i}$.

Raman maps presented in the manuscript were constructed based on principles of Cluster Analysis described above. Number of clusters was 6 (the minimum number of clusters characterized by different average Raman spectra, which describe the variety of the inhomogeneous biological sample). The colors of the clusters correspond to the colors of the average Raman spectra of collagen (red), Cyt $c$ (green), oleic acid and $\beta$-carotene (blue), palmitic acid (pink), mammaglobin-A (yellow), cardiolipin (turquoise).

Basis analysis. The Basis analysis was performed based on the Raman spectra recorded for pure collagen, cytochrome $c$, oleic acid and $\beta$-carotene, palmitic acid, mammaglobin-A, and cardiolipin. During the analysis each measured spectrum of the $2 \mathrm{D}$ spectral array of the analyzed human breast sample was compared to the spectra of pure chemical components mentioned above using a least square to fit each convergence to minimize the fitting error $\mathrm{D}$ described by equation:

$$
D=\left([\overrightarrow{\text { Recorded spectrum }}]-a \times \overrightarrow{B S_{\mathrm{A}}}-b \times \overrightarrow{B S_{\mathrm{B}}}-c \times \overrightarrow{B S_{\mathrm{C}}}-\cdots\right)^{2}
$$

by varying the weighting factors $a, b, c, \ldots$ of the basis spectra $\overrightarrow{B S}$.

Received: 10 June 2021; Accepted: 29 December 2021

Published online: 08 February 2022 


\section{References}

1. García-Heredia, J. M. et al. Tyrosine phosphorylation turns alkaline transition into a biologically relevant process and makes human cytochrome $c$ behave as an anti-apoptotic switch. J. Biol. Inorg. Chem. 16, 1155-1168 (2011).

2. Eleftheriadis, T., Pissas, G., Liakopoulos, V. \& Stefanidis, I. Cytochrome $c$ as a potentially clinical useful marker of mitochondrial and cellular damage. Front. Immunol. 7, 279 (2016).

3. Kogot-Levin, A. et al. Upregulation of mitochondrial content in cytochrome $c$ oxidase deficient fibroblasts. PLoS ONE 11, e0165417 (2016).

4. Vaughn, A. E. \& Deshmukh, M. Glucose metabolism inhibits apoptosis in neurons and cancer cells by redox inactivation of cytochrome c. Nat. Cell Biol. 10, 1477-1483 (2008).

5. Gogvadze, V., Orrenius, S. \& Zhivotovsky, B. Multiple pathways of cytochrome $c$ release from mitochondria in apoptosis. Biochim. Biophys. Acta Bioenerg. 1757, 639-647 (2006).

6. Ow, Y. L. P., Green, D. R., Hao, Z. \& Mak, T. W. Cytochrome c: Functions beyond respiration. Nat. Rev. Mol. Cell Biol. 9, 532-542 (2008).

7. Santidrian, A. F. et al. Mitochondrial complex I activity and NAD+/NADH balance regulate breast cancer progression. J. Clin. Invest. 123, 1068-1081 (2013).

8. Ordys, B. B., Launay, S., Deighton, R. F., McCulloch, J. \& Whittle, I. R. The role of mitochondria in glioma pathophysiology. Mol. Neurobiol. 42, 64-75 (2010).

9. Grupp, K. et al. High mitochondria content is associated with prostate cancer disease progression. Mol. Cancer 12, 145 (2013).

10. Chishiki, M. et al. Cytochrome $c 1$ in ductal carcinoma in situ of breast associated with proliferation and comedo necrosis. Cancer Sci. 108, 1510-1519(2017).

11. Liu, Z., Zhao, X., Zhang, L. \& Pei, B. Cytochrome $c$ inhibits tumor growth and predicts favorable prognosis in clear cell renal cell carcinoma. Oncol. Lett. 18, 6026-6032 (2019).

12. Pelicano, H. et al. Mitochondrial dysfunction in some triple-negative breast cancer cell lines: Role of mTOR pathway and therapeutic potential. Breast Cancer Res. 16, 434 (2014).

13. Wallace, D. C. Mitochondria and cancer. Nat. Rev. Cancer 12, 685-698 (2012).

14. Liu, X., Kim, C. N., Yang, J., Jemmerson, R. \& Wang, X. Induction of apoptotic program in cell-free extracts: Requirement for dATP and cytochrome $c$. Cell 86, 147-157 (1996).

15. Wang, C. \& Youle, R. J. The role of mitochondria in apoptosis. Annu. Rev. Genet. 43, 95-118 (2009).

16. Ricci, J.-E. et al. Disruption of mitochondrial function during apoptosis is mediated by caspase cleavage of the p75 subunit of complex I of the electron transport chain. Cell 117, 773-786 (2004).

17. Garrido, C. et al. Mechanisms of cytochrome $c$ release from mitochondria. Cell Death Differ. 13, 1423-1433 (2006).

18. Manickam, P., Kaushik, A., Karunakaran, C. \& Bhansali, S. Recent advances in cytochrome $c$ biosensing technologies. Biosens. Bioelectron. 87, 654-668 (2017).

19. Movasaghi, Z., Rehman, S. \& Rehman, I. U. Raman spectroscopy of biological tissues. Appl. Spectrosc. Rev. 42, 493-541 (2007).

20. Koljenović, S., Schut, T. B., Vincent, A., Kros, J. M. \& Puppels, G. J. Detection of meningioma in dura mater by Raman spectroscopy. Anal. Chem. 77, 7958-7965 (2005).

21. Shetty, G., Kendall, C., Shepherd, N., Stone, N. \& Barr, H. Raman spectroscopy: Elucidation of biochemical changes in carcinogenesis of oesophagus. Br. J. Cancer 94, 1460-1464 (2006).

22. Abramczyk, H. et al. Redox imbalance and biochemical changes in cancer by probing redox-sensitive mitochondrial cytochromes in label-free visible resonance raman imaging. Cancers 13, 960 (2021).

23. Brown, G. C. \& Borutaite, V. Regulation of apoptosis by the redox state of cytochrome c. Biochim. Biophys. Acta Bioenerg. 1777, 877-881 (2008).

24. Kagan, V. E. et al. Oxidative lipidomics of apoptosis: Redox catalytic interactions of cytochrome $c$ with cardiolipin and phosphatidylserine. Free Radic. Biol. Med. 37, 1963-1985 (2004).

25. Abramczyk, H., Brozek-Pluska, B., Surmacki, J., Tondusson, M. \& Freysz, E. Photostability of biological systems-Femtosecond dynamics of zinc tetrasulfonated phthalocyanine at cancerous and noncancerous human breast tissues. J. Photochem. Photobiol. A Chem. 332, 10-24 (2017).

26. Ripple, M. O., Abajian, M. \& Springett, R. Cytochrome $c$ is rapidly reduced in the cytosol after mitochondrial outer membrane permeabilization. Apoptosis 15, 563-573 (2010).

27. Ntambi, J. M. Regulation of stearoyl-CoA desaturase by polyunsaturated fatty acids and cholesterol. J. Lipid Res. 40, 1549-1558 (1999).

28. Heimburg, T. Thermal Biophysics of Membranes. Thermal Biophysics of Membranes (Wiley, 2007). https://doi.org/10.1002/97835 27611591

29. Unsay, J. D., Cosentino, K., Subburaj, Y. \& García-Sáez, A. J. Cardiolipin effects on membrane structure and dynamics. Langmuir 29, 15878-15887 (2013).

30. Zhao, W., Tisza, M., Sjol, J., Mani, S. \& Chang, J. T. Abstract 2080: Plasma membrane fluidity drives metastasis in breast cancer. Tumor Biol. 74, 2080-2080 (2014).

31. Ignatiadis, M. et al. Prognostic value of the molecular detection of circulating tumor cells using a multimarker reverse transcriptionPCR assay for cytokeratin 19, mammaglobin A, and HER2 in early breast cancer. Clin. Cancer Res. 14, 2593-2600 (2008).

32. Deberardinis, R. J. \& Thompson, C. B. Cellular metabolism and disease: What do metabolic outliers teach us?. Cell 148, 1132-1144 (2012).

33. Qu, W. et al. Preparation and characterization of L-[5-11C]-glutamine for metabolic imaging of tumors. J. Nucl. Med. 53, 98-105 (2012).

34. Don, A. S., Lim, X. Y. \& Couttas, T. A. Re-configuration of sphingolipid metabolism by oncogenic transformation. Biomolecules 4, 315-353 (2014)

35. Tang, X. et al. A joint analysis of metabolomics and genetics of breast cancer. Breast Cancer Res. 16, 415 (2014).

36. Muñoz-Pinedo, C., El Mjiyad, N. \& Ricci, J. E. Cancer metabolism: Current perspectives and future directions. Cell Death Dis. 3, e248 (2012).

37. Santos, C. R. \& Schulze, A. Lipid metabolism in cancer. FEBS J. 279, 2610-2623 (2012).

38. Baenke, F., Peck, B., Miess, H. \& Schulze, A. Hooked on fat: The role of lipid synthesis in cancer metabolism and tumour development. DMM Dis. Model. Mech. 6, 1353-1363 (2013).

39. Currie, E., Schulze, A., Zechner, R., Walther, T. C. \& Farese, R. V. Cellular fatty acid metabolism and cancer. Cell Metab. 18, 153-161 (2013).

40. Baumann, J., Sevinsky, C. \& Conklin, D. S. Lipid biology of breast cancer. Biochim. Biophys. Acta Mol. Cell Biol. Lipids 1831, 1509-1517 (2013).

41. Menendez, J. A. \& Lupu, R. Fatty acid synthase and the lipogenic phenotype in cancer pathogenesis. Nat. Rev. Cancer 7, 763-777 (2007).

42. Guo, D., Bell, E. H. \& Chakravarti, A. Lipid metabolism emerges as a promising target for malignant glioma therapy. CNS Oncol. 2, 289-299 (2013) 
43. Abramczyk, H., Imiela, A. \& Śliwińska, A. Novel strategies of Raman imaging for exploring cancer lipid reprogramming. J. Mol. Liq. 274, 52-59 (2019).

44. Surmacki, J., Brozek-Pluska, B., Kordek, R. \& Abramczyk, H. The lipid-reactive oxygen species phenotype of breast cancer. Raman spectroscopy and mapping, PCA and PLSDA for invasive ductal carcinoma and invasive lobular carcinoma. Molecular tumorigenic mechanisms beyond Warburg effect. Analyst 140, 2121-2133 (2015).

45. Abramczyk, H., Surmacki, J. M., Brozek-Pluska, B. \& Kopec, M. Revision of commonly accepted Warburg mechanism of cancer development: Redox-sensitive mitochondrial cytochromes in breast and brain cancers by Raman imaging. Cancers 13, 2599 (2021).

\section{Acknowledgements}

This work was supported by NCN Poland Grant UMO-2019/33/B/ST4/01961.

\section{Author contributions}

Conceptualization: H.A.; Funding acquisition: H.A.; Investigation: B.B.-P., M.K.; Methodology: H.A., B.B.-P., M.K., Writing-original draft: H.A.; Manuscript editing: H.A., B.B.-P., M.K. All authors have read and agreed to the published version of the manuscript.

\section{Competing interests}

The authors declare no competing interests.

\section{Additional information}

Supplementary Information The online version contains supplementary material available at https://doi.org/ 10.1038/s41598-022-04803-0.

Correspondence and requests for materials should be addressed to H.A.

Reprints and permissions information is available at www.nature.com/reprints.

Publisher's note Springer Nature remains neutral with regard to jurisdictional claims in published maps and institutional affiliations.

(c) (i) Open Access This article is licensed under a Creative Commons Attribution 4.0 International License, which permits use, sharing, adaptation, distribution and reproduction in any medium or format, as long as you give appropriate credit to the original author(s) and the source, provide a link to the Creative Commons licence, and indicate if changes were made. The images or other third party material in this article are included in the article's Creative Commons licence, unless indicated otherwise in a credit line to the material. If material is not included in the article's Creative Commons licence and your intended use is not permitted by statutory regulation or exceeds the permitted use, you will need to obtain permission directly from the copyright holder. To view a copy of this licence, visit http://creativecommons.org/licenses/by/4.0/.

(c) The Author(s) 2022 